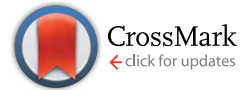

Cite this: J. Mater. Chem. A, 2016, 4, 6955

Received 18th December 2015 Accepted 2nd February 2016

DOI: $10.1039 / \mathrm{c} 5 \operatorname{ta} 10401 \mathrm{~g}$

www.rsc.org/MaterialsA

\section{Amino acids as highly efficient modulators for single crystals of zirconium and hafnium metal- organic frameworks $\uparrow$}

\author{
Ross J. Marshall, ${ }^{a}$ Claire L. Hobday, ${ }^{b}$ Colin F. Murphie, ${ }^{a}$ Sarah L. Griffin, ${ }^{a}$

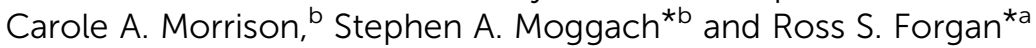

\begin{abstract}
The synthesis of zirconium and hafnium metal-organic frameworks (MOFs) often relies on coordination modulation - the addition of competing monotopic modulators to reaction mixtures - to reproducibly generate highly crystalline material. Typically, large excesses of monocarboxylic acids such as formic, acetic and benzoic acid are applied, but access to diffraction quality single crystals, particularly of UiO66 topology MOFs, remains troublesome. Herein, we show that amino acids, in particular L-proline, are highly efficient modulators of $\mathrm{Zr}$ and $\mathrm{Hf}$ MOFs of the $\mathrm{UiO}-66$ series, with as little as four equivalents affording access to large, diffraction quality single crystals that are free of defects. Five crystal structures are reported, including MOFs which previously could not be characterised in this manner, with molecular dynamics simulations utilised to understand dynamic disorder. Additionally, a series of MOFs are characterised in depth, allowing a comparison of the thermal stabilities and porosities for $\mathrm{Zr}$ and $\mathrm{Hf}$ analogues. We also show that the protocol can be extended to microwave synthesis, and that modulating ability varies dramatically across a series of amino acids. Access to single crystals has facilitated our own in depth study of the mechanical properties of these MOFs, and we expect that our protocols will enable the discovery of new $\mathrm{Zr}$ and Hf MOFs as well as offer new insights into their materials properties.
\end{abstract}

\section{Introduction}

Metal-organic frameworks (MOFs) are 3D hybrid materials that have received appreciable amounts of interest in recent years ${ }^{1}$ as the almost infinite combination of inorganic metal clusters and organic spacing ligands achievable allows highly specialised materials to be investigated for use in a wide range of applications including selective gas capture and sequestration, ${ }^{2}$ drug delivery ${ }^{3}$ and catalysis. ${ }^{4}$ Some MOFs have been known to exhibit relatively low chemical and mechanical stabilities, but the use of zirconium, a strong Lewis acid that demonstrates a high affinity for oxygen, results in resilient MOFs as a result of the robust $\mathrm{Zr}-\mathrm{O}$ bonds that tether the organic and inorganic moieties. ${ }^{5}$

${ }^{a}$ WestCHEM School of Chemistry, University of Glasgow, Joseph Black Building, University Avenue, Glasgow, G12 8QQ, UK. E-mail: Ross.Forgan@glasgow.ac.uk; Web: http://www.forganlab.com

${ }^{b}$ EaSTCHEM School of Chemistry, University of Edinburgh, Joseph Black Building, David Brewster Road, Edinburgh, EH9 3FJ, UK. E-mail: s.moggach@ed.ac.uk; Web: http://www.moggach.chem.ed.ac.uk

$\dagger$ Electronic supplementary information (ESI) available. CCDC 1442841-1442842. For ESI and crystallographic data in CIF or other electronic format see DOI: $10.1039 / \mathrm{c} 5$ ta10401g
Many zirconium MOFs adopt the well-documented UiO-66 framework topology containing $\mathrm{Zr}_{6} \mathrm{O}_{4}(\mathrm{OH})_{4}$ secondary building units (SBUs) connected by 12 bridging dicarboxylate ligands, thereby expanding to form cubic framework structures containing large octahedral cages and smaller tetrahedral cages. ${ }^{5 \boldsymbol{b}}$ Incorporation of organic ligands that present different coordination environments, including tri- and tetra-topic ligands, into Zr MOFs leads to materials with alternative structures and topologies. ${ }^{6}$ Functionalised ligands presenting greater chemical complexity, or bearing a functional group required for specific post-synthetic modification, have been incorporated in the anticipation of producing highly specialised materials. ${ }^{\boldsymbol{6} \boldsymbol{b}, \boldsymbol{d}, \boldsymbol{e}, \boldsymbol{7}}$ Hafnium displays similar chemical properties to zirconium; hafnium MOFs are isostructural with their zirconium analogues, where direct substitution of the $\mathrm{Zr}_{6}$ SBUs by $\mathrm{Hf}_{6}$ ones is observed. ${ }^{8}$ There are fewer reports of hafnium MOFs in the literature, ${ }^{\mathbf{6}, \mathbf{9}}$ but they have shown great promise in medicinal applications, ${ }^{10}$ as well as in catalysis ${ }^{11}$ and $\mathrm{CO}_{2}$ fixation. ${ }^{12}$

Coordination modulation ${ }^{13}$ results in materials with enhanced crystallinity and has recently enabled the isolation of single crystals of zirconium and hafnium MOFs with the UiO-66 topology. ${ }^{6 \boldsymbol{b}, \boldsymbol{d}, \boldsymbol{e}, \boldsymbol{c} \boldsymbol{c}, \boldsymbol{d}, \mathbf{9 b}, \mathbf{1 4}}$ This technique usually requires the addition of large excesses of small foreign materials directly to the MOF synthesis, with small monodentate organic compounds, such as acetic acid and benzoic acid, routinely employed. The 
precise role of the modulator during synthesis is not known, although it is believed that the crystallisation kinetics are altered, introducing reversibility to the system and ultimately resulting in products with improved crystallinity. ${ }^{15}$ Preformation of $\mathrm{Zr}_{6} \mathrm{O}_{4}(\mathrm{OH})_{4}\left(\mathrm{RCO}_{2}\right)_{12}$ building blocks containing the monodentate modulators may also be responsible for the enhanced crystallinity of the resulting MOFs. ${ }^{14 b}$ Under certain conditions, modulation can also promote defect formation, which is prevalent ${ }^{\mathbf{9 d}, \mathbf{1 4 f , 1 6}}$ in UiO-66 type MOFs of both $\mathrm{Zr}$ and $\mathrm{Hf}$, and these defects can be exploited to confer the MOFs with enhanced gas uptake, ${ }^{17}$ proton conduction, ${ }^{18}$ mechanical ${ }^{5 f}$ or catalytic ${ }^{19}$ properties.

Recently the scope of modulators has been broadened and the role of hydrochloric acid in zirconium MOF synthesis has been investigated. $\mathrm{HCl}$ modulation was observed to increase the rate of product formation whilst providing access to otherwise inaccessible materials, therefore it has been postulated that $\mathrm{HCl}$ could perform numerous roles during synthesis, including conditioning the DMF solvent or assisting in the pre-formation of $\mathrm{Zr}_{6}$ clusters. ${ }^{16 \boldsymbol{b}, 20}$ Despite these advances, reliable isolation of X-ray quality single crystals of UiO-66 topology Zr and Hf MOFs remains troublesome, and so we seek to extend the range of potential modulators for both bulk and single crystal syntheses of $\mathrm{Zr}$ and Hf MOFs.

\section{Results and discussion}

Herein, we fully investigate for the first time the ability of amino acids to act as modulating agents in the directed synthesis of both zirconium and hafnium MOFs. Amino acids can be viewed as attractive modulating agents as they are readily available small biomolecules and the large diversity of side-chains within these compounds may potentially result in unprecedented modulating properties. We have investigated ${ }^{21}$ a comprehensive range of ligands (L1-L8, Scheme 1) and report the influence of seven amino acids on the crystallinity of the products obtained, which have the general formula $\left[\mathrm{M}_{6} \mathrm{O}_{4}(\mathrm{OH})_{4} \mathrm{~L}_{6}\right]_{n}$,

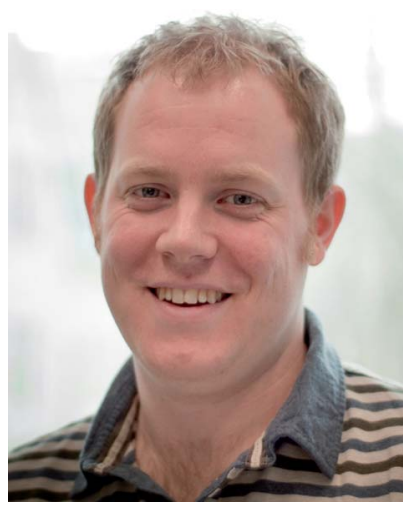

Ross Forgan is a Royal Society University Research Fellow in the School of Chemistry at the University of Glasgow. After receiving MChem (Hons) and $\mathrm{PhD}$ degrees from the University of Edinburgh in 2004 and 2008, under the supervision of Prof. Peter Tasker, he spent three years as a postdoctoral researcher in the group of Prof. Sir J. Fraser Stoddart at Northwestern University, USA. In 2011 he returned to Scotland as a postdoctoral researcher in the group of Prof. Lee Cronin at the University of Glasgow, and took up his current independent position in 2012. His research group is interested in the crystallisation and functionalisation of MOFs, as well as their materials properties and biomimetic applications.

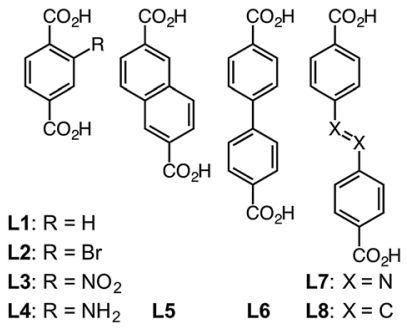

Scheme 1 Chemical structures of the dicarboxylate ligands, L1-L8, utilised in this study.

where $\mathbf{M}=\mathrm{Zr}^{4+}$ or $\mathrm{Hf}^{4+}$, and are referred to as $\mathbf{M}-\mathbf{L}$ for simplicity. MOF syntheses were carried out to a general synthetic protocol (see ESI, Section S2 $\dagger$ ). One equivalent each of $\mathrm{MCl}_{4}$ and the chosen ligand were dissolved separately in reagent grade $N, N$-dimethylformamide (DMF), with five equivalents of the amino acid and one equivalent of $\mathrm{HCl}$ added to the metal solution. The solutions were combined (the final concentrations of metal and ligand being $45 \mathrm{mM}$ ), sonicated and heated for $24 \mathrm{~h}$ at $120{ }^{\circ} \mathrm{C}$. The resultant precipitates were thoroughly washed by DMF and methanol, and vacuum dried. ${ }^{22}$ Powder $\mathrm{X}$-ray diffraction (PXRD) analysis was used to determine the extent of formation and crystallinity of the MOFs in comparison to identical control samples with no amino acid added.

Our examination of this broad experimental parameter space showed dramatic improvements in crystallinity of the Zr MOFs of the longer linkers, L5-L8, when certain amino acids were used as modulators; the results for Zr-L5 (Fig. 1) are representative of the longer linkers. The single crystal structure of Zr-L5 has been reported ${ }^{9 b}$ previously, using 47 equivalents of acetic

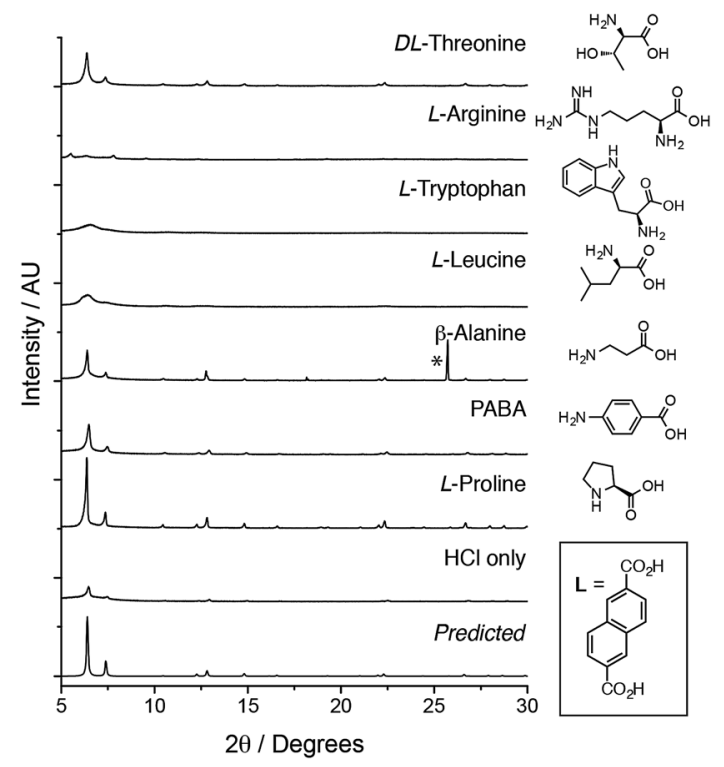

Fig. 1 Stacked PXRD patterns of Zr-L5 synthesised with different amino acid modulators (five equivalents), compared with Zr-L5 synthesised with one equivalent of $\mathrm{HCl}$ only and the pattern predicted from the previously reported crystal structure. ${ }^{9 b}$ An unknown crystalline impurity, marked by an asterisk, was occasionally found in $\beta$ alanine modulated samples. 
acid as modulator, and comparison of the PXRD patterns of the amino acid modulated syntheses with that predicted from the crystal structure confirm that Zr-L5 is formed cleanly, and that the smaller, more hydrophobic amino acids, in concert with $\mathrm{HCl}$, significantly enhance the crystallinity of the product in comparison to syntheses using $\mathrm{HCl}$ alone. ${ }^{23}$

Across the Zr MOFs of L5-L8, L-proline stands out as the most effective modulator, with considerable improvements in the crystallinity of the MOFs (Fig. 2a). SEM images of materials synthesised according to the general synthetic procedure using five equivalents of L-proline (Fig. 2b and d) show Zr-L5 and Zr-L7 to form sheets of polycrystalline material, which has been observed previously ${ }^{24}$ for Zr-L7, with intergrown crystals of around 5-10 $\mu \mathrm{m}$ for Zr-L5 and up to $50 \mu \mathrm{m}$ for Zr-L7. In contrast, Zr-L6 and Zr-L8 tend to form discrete octahedral crystals (Fig. 2c and e), around 10-20 $\mu \mathrm{m}$ in size for Zr-L6 (but with occasional larger crystals up to $60 \mu \mathrm{m}$ ) and 5-10 $\mu \mathrm{m}$ size for Zr-L8, which also forms some spherical polycrystalline assemblies (see ESI, Section $\mathrm{S} 3 \dagger$ ).

In contrast, the synthesis of Zr MOFs of L1-L4, all terephthalate-based linkers, did not show any appreciable benefits when amino acid modulation was employed in comparison with samples prepared using $\mathrm{HCl}$ alone. The binding of monocarboxylic acids to the zirconium cluster in situ has been proposed to decelerate the rate of crystallisation, through establishing exchange equilibrium with the bridging organic ligands, ${ }^{\mathbf{1 5}}$ and so we are unable to account for this unusual, seemingly size-selective phenomenon.

The overwhelming performance exhibited by L-proline directed future modulation studies of zirconium MOFs. Considering our synthetic technique combines $\mathrm{HCl}$ and L-proline it was vital to investigate the modulation ability of $\mathrm{L}$-proline in the presence/absence of $\mathrm{HCl}$, considering the recent work detailing the advantages of addition of $\mathrm{HCl}$ to zirconium MOF syntheses. ${ }^{16 \boldsymbol{b}, 20} \mathbf{Z r - L 6}$ (UiO-67) was used to demonstrate the effect of L-proline modulation (see ESI, Section S4†). PXRD patterns (Fig. 3a) showed that while addition of one equivalent of $\mathrm{HCl}$ alone to solvothermal syntheses was capable of intermittently producing relatively crystalline material, ${ }^{23}$ addition of five equivalents of L-proline consistently produced better quality materials, and a synergistic effect appears to be present when both are incorporated into syntheses. Proline equivalency studies on Zr-L6 (see ESI Section S4 $\dagger$ ) revealed that the addition of between 4 and 10 equivalents of L-proline to syntheses alongside $\mathrm{HCl}$ resulted in significantly improved PXRD patterns (Fig. 3b). SEM images of the synthesised materials (Fig. 3c) showed that when two equivalents of L-proline are added to the synthesis, the Zr-L6 that forms is ill-defined with a globular morphology, while samples with between four and ten equivalents of L-proline added to syntheses resulted in well-defined octahedral crystals around $10-20 \mu \mathrm{m}$ in size.

Examination of both PXRD patterns and SEM images suggest that four to five equivalents of $\mathrm{L}_{\text {-proline and one equivalent of }}$ $\mathrm{HCl}$ represent the most efficient modulating conditions. Syntheses can also be carried out under microwave heating, ${ }^{25}$ with reduced synthesis times (see ESI, Section S5 $\dagger$ ). Zr-L6 prepared in $1 \mathrm{~h}$ in a single mode microwave cavity, using five equivalents of L-proline, showed comparable particle size, morphology and porosity to samples prepared by heating for $24 \mathrm{~h}$ in the oven.

Further optimisation of the L-proline/HCl modulated conditions allowed the synthesis (see ESI, Section S6 $\dagger$ ) of single crystals of many of the MOFs (Fig. 4a-c). High quality single crystals of Zr-L6, approximately $60 \mu \mathrm{m}$ in size, were synthesised solvothermally, at $120{ }^{\circ} \mathrm{C}$ in DMF for $24 \mathrm{~h}$, in the presence of 5 equivalents of L-proline and one equivalent of $\mathrm{HCl}$, which is minimal when compared to the large excess of modulators commonly required for effective modulation. Until recently, Zr-L6 had only been isolated as a microcrystalline powder however, during the course of this study single crystals were reported by a number of different groups. Kaskel et al. synthesised the MOF solvothermally, using 117 equivalents of acetic acid modulator, ${ }^{26}$ Lillerud et al. used 30 equivalents of benzoic acid and one equivalent of $\mathrm{HCl}$ as modulators in solvothermal syntheses using glassware first pre-treated with basic aqueous solution, ${ }^{27} \mathrm{Kim}$ et al. reported a synthesis modulated
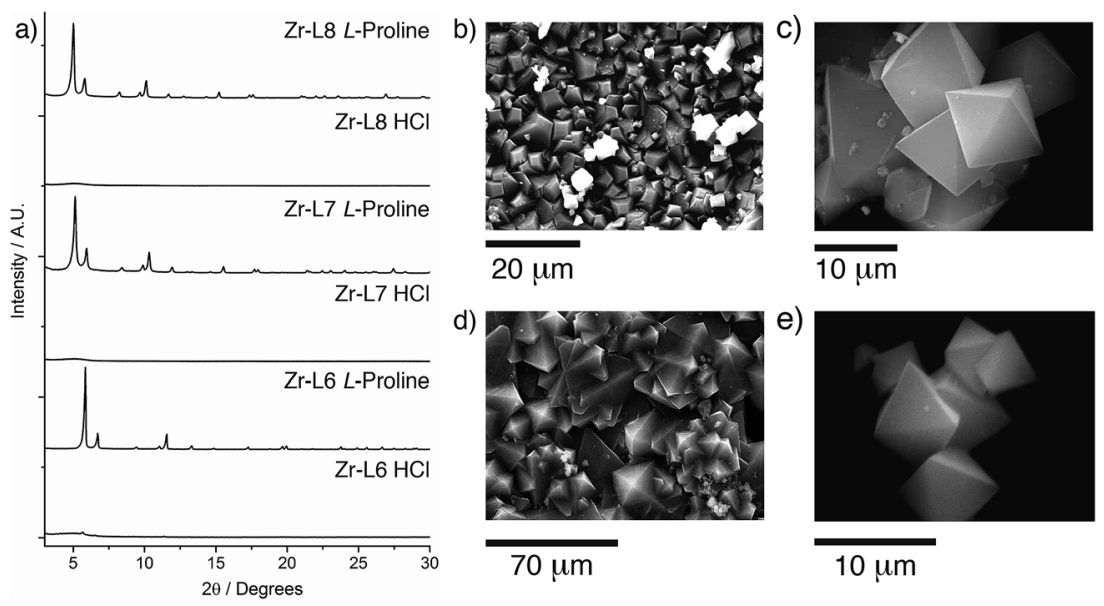

Fig. 2 (a) PXRD patterns showing the effects of incorporation of 5 equivalents of $L$-proline and one of $\mathrm{HCl}$ into solvothermal syntheses of $\mathrm{Zr}$ MOFs of L6-L8 compared to addition of HCl alone. SEM images of the MOFs formed using this protocol: (b) Zr-L5, (c) Zr-L6, (d) Zr-L7, (e) Zr-L8. 

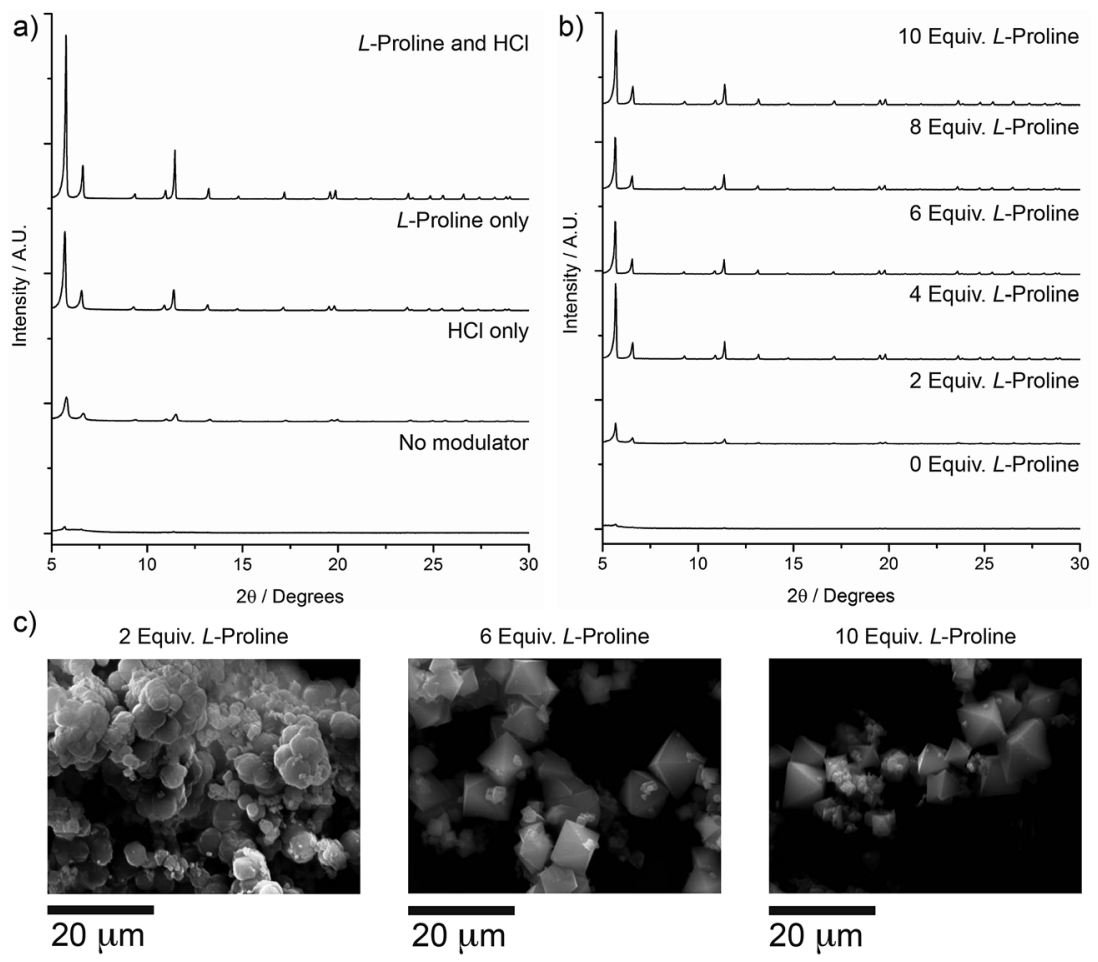

Fig. 3 (a) PXRD patterns of syntheses of Zr-L6 with differing modulators, showing that L-proline (5 equivalents) is an effective modulator alone but seems to give best results in concert with $\mathrm{HCl}$ (one equivalent). (b) PXRD patterns showing the effect of varying the amount of $\mathrm{L}$-proline added to the syntheses of $\mathrm{Zr}-\mathrm{L} 6$ which also contain $\mathrm{HCl}$, showing little improvement after more than four equivalents are used. (c) SEM images show that Zr-L6 synthesised using two equivalents of L-proline produce an ill-defined material, while addition of either six or ten equivalents yields octahedral crystals around $10 \mu \mathrm{m}$ in size.

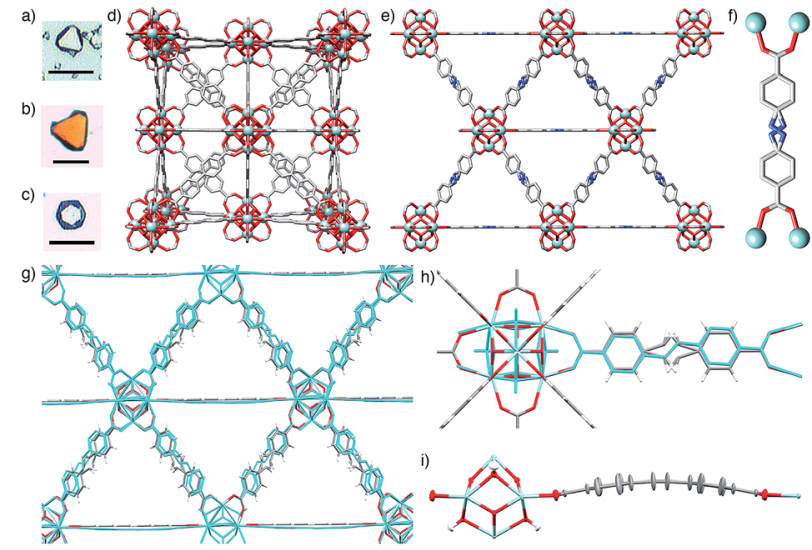

Fig. 4 Pictures of single crystals of (a) Zr-L6, (b) Zr-L7, and (c) Zr-L8 prepared by L-proline modulation. Scale bars $100 \mu \mathrm{m}$. (d) Packing structure of Zr-L6 viewed down the crystallographic a axis. (e) Packing structure of $\mathrm{Zr}-\mathrm{L} 7$ from a different angle. (f) Disorder in the geometrically frustrated azobenzene ligand in Zr-L7. Overlay of closely correlated calculated (teal, MD simulations) and experimental structures of $\mathbf{Z r - L 8}$, which is isostructural with $\mathrm{Zr}$-L7, viewed (g) in a packing arrangement, and (h) zoomed in onto a single L8 linker, showing the simulation closely matches the observed disorder. (i) Atomic pairdistribution function derived from the MD trajectory showing large thermal motion in the bent L8 linker in Zr-L8. by 30 equivalents of benzoic acid, ${ }^{28}$ while Shafir and Gutov reported a similar benzoic acid (30 equivalents) modulated route which included a subsequent "healing" step using additional L6. Our own determination of the crystal structure of Zr-L6 showed it to be very similar to those reported recently (Fig. 4d), with no evidence of defects or diffuse scattering observed. ${ }^{29}$

Similarly, L-proline modulation yielded $50-100 \mu \mathrm{m}$ single crystals of both Zr-L7 and Zr-L8, which have "bent" linkers. ZrL7 was reported previously in 2012 by Behrens et al., with the use of 30 equivalents of benzoic acid as the modulator resulting in crystalline sheets rather than discrete single crystals, and weak diffraction combined with disorder meaning no crystal structure could be elucidated. ${ }^{24} \mathrm{~A}$ recent catalytic study reported an acetic acid (525 equivalents) modulated synthesis of Zr-L7 that resulted in micron-sized particles. ${ }^{30}$ Isolation of discrete octahedral single crystals of $\mathbf{Z r - L 7}$ and $\mathbf{Z r}-\mathbf{L} \mathbf{8}$ was possible using four equivalents of $\mathrm{L}$-proline and one equivalent of $\mathrm{HCl}$ in a solvothermal synthesis at $100{ }^{\circ} \mathrm{C}$ for $48 \mathrm{~h}$ in DMF. During repeated syntheses, it was noted that order of addition was key to gaining large single crystals; addition of $\mathrm{HCl}$ to a pre-sonicated mixture of the other reagents provided optimal results.

Determination of the crystal structures of the closely related Zr-L7 and Zr-L8 MOFs revealed them to be isostructural (Fig. 4e). Despite the non-linearity of the "bent" azobenzene and stilbene linkers, $\mathbf{L} 7$ and $\mathbf{L 8}$, the resultant MOFs crystallise in the cubic $F m \overline{3} m$ space group, with the ligands disordered at their 
interiors and exhibiting geometric frustration (Fig. 4f). The longer linkers result in larger unit cells; for $\mathbf{Z r - L 7}, a=29.3248(8)$ $\AA$ and for Zr-L8, the larger value of $a=29.8884$ (3) Å may be due to the longer length of the $\mathrm{C}=\mathrm{C}$ bond compared to the $\mathrm{N}=\mathrm{N}$ bond. ${ }^{31}$ Indeed, the separation between the $\mathrm{Zr}_{6}$ metal clusters, measured from analogous carboxylate oxygen atoms on an individual linker molecule, increases from 13.3104(2) $\AA$ in Zr-L7 to $13.5713(1) \AA$ in $\mathbf{Z r - L 8}$, illustrating the increase in linker length.

Molecular dynamics (MD) simulations were utilised to understand the nature of the disorder in the MOFs and also to elucidate the slight differences in unit cell values (see ESI, Section S7 $\dagger$ ). We have previously used MD simulations to characterise the structural properties of Zr-L7 in a communication discussing its mechanical properties, ${ }^{21}$ and so analogous calculations were carried out on Zr-L8. The simulations confirm that the increase in unit cell length from Zr-L7 to Zr-L8 is a consequence of moving from an azobenzene to a stilbene linker, and reveal that the geometrically frustrated ligands, $\mathbf{L 7}$ and $\mathbf{L 8}$, are accommodated in the structures by bowing out of the mirror plane, sitting above and below the plane with bowing angles as high as $10^{\circ}$ (Fig. 4g, average value $5(3)^{\circ}$, see ESI, Section S7 $\dagger$ ). The average effect of this dynamic disorder process, wherein the linkers bend and flip between opposite sides of the mirror plane, is the frustrated linear arrangement as observed in the crystal structure.

The time-averaged structures generated by these simulations show a close correlation to the spatially-averaged disorder in the experimental crystal structures (Fig. 4h), suggesting that the flexing of the ligand is responsible for the disorder, and atomic pair-distribution functions derived from the MD trajectory show that the ligand is indeed the most dynamic component of the structure (Fig. 4i). Finally, key bond distances within the $\mathrm{Zr}_{6}$ clusters of Zr-L8 match closely to those of its solid-state structure and those calculated for UiO-66 (Zr-L1) by Lamberti et al. ${ }^{16 d}$ validating the simulations. The disorder model applied to the structures of Zr-L7 and Zr-L8 is very representative of the bulk material; PXRD patterns predicted from the single crystal structures match the experimental patterns from bulk-synthesised samples closely (see ESI, Section S7 $†$ ).

Following effective amino acid modulation of the zirconium MOFs, we investigated the suitability of this technique for the synthesis of their hafnium analogues (see ESI, Section S8†). We initially sought to investigate the effect of both $\mathrm{HCl}$ and L-proline inclusion during the synthesis of Hf-L6, analogous to the control experiments performed for Zr-L6 (Fig. 5a). Surprisingly, $\mathrm{HCl}$ alone proved to be an ineffective modulator for synthesis of Hf-L6, even in the quantities found to modulate the synthesis of Zr-L6 by Farha et al., ${ }^{16 b}$ however L-proline is observed to greatly enhance crystallinity. As with Zr-L6, it was observed that a combination of one equivalent of $\mathrm{HCl}$ and five equivalents of L-proline in the synthetic mixture leads to the greatest improvement in crystallinity of Hf-L6, yielding 10-20 $\mu \mathrm{m}$ octahedral crystals instead of amorphous material (Fig. 5b and c). Subsequently, syntheses of Hf-MOFs of L1-L8 were attempted using $\mathrm{HfCl}_{4}$ as a replacement for $\mathrm{ZrCl}_{4}$ in the reaction mixture, and modulated by six different amino acids. ${ }^{32}$ As with the zirconium analogues, the greatest enhancement in crystallinity from amino acid modulation is observed for the Hf MOFs of the longer linkers, L5-L8, although para-aminobenzoic acid seems to slightly improve the PXRD patterns of the Hf MOFs of the terephthalate ligands, L1-L4, when it is used as the

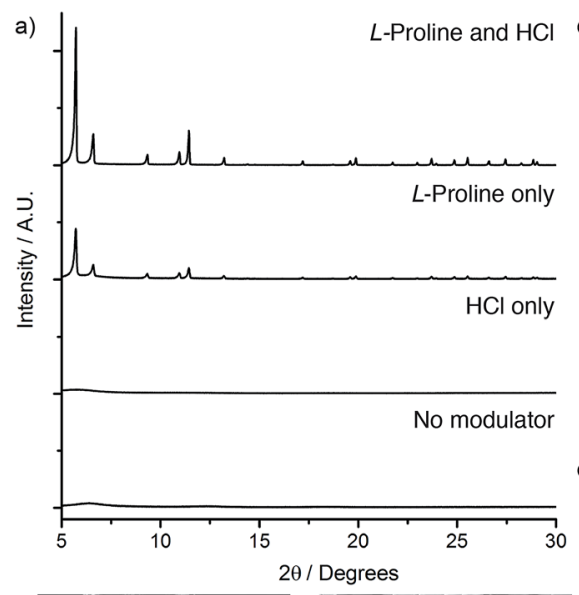

d)
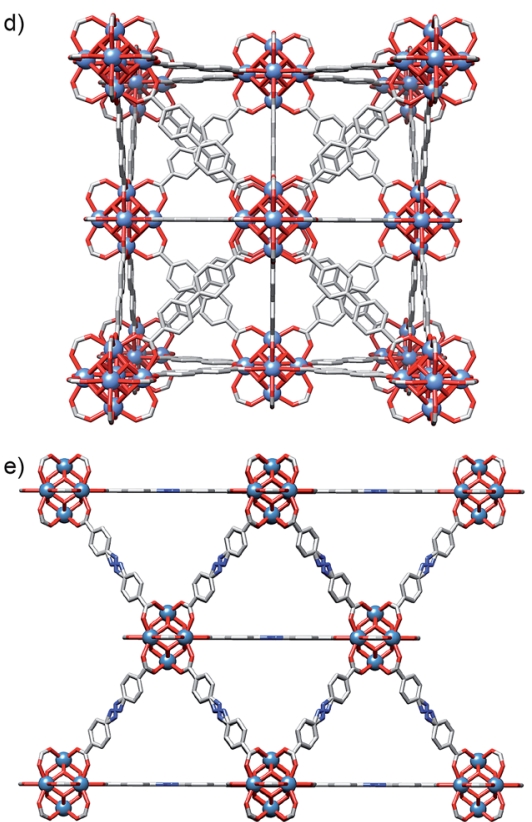

Fig. 5 (a) Stacked PXRD patterns showing the crystallinity of $\mathrm{Hf}-\mathrm{L} 6$ is enhanced by L-proline modulation, particularly in concert with $\mathrm{HCl}$, which does not give crystalline material when used alone. SEM images of the products of (b) unmodulated synthesis and (c) L-proline and $\mathrm{HCl}$ modulated synthesis of Hf-L6. (d) Packing structure derived from the crystal structure of Hf-L6. (e) Packing structure derived from the crystal structure of Hf-L7, with disordered azobenzene linkers. Disorder in phenyl ring orientations not shown. 
Table 1 A comparison of some crystallographic parameters for the $\mathrm{Zr}$ and Hf MOFs characterised by single crystal X-ray diffraction in this study

\begin{tabular}{|c|c|c|c|c|}
\hline MOF & Unit cell edge $/ \AA$ & Unit cell Vol/ $/ \AA^{3}$ & M-OOC bond/Å & $\mathbf{M}_{6}$ separation $/ \AA$ \\
\hline Zr-L6 & $26.8564(5)$ & $19370.6(11)$ & $2.227(3)$ & $11.209(4)$ \\
\hline Hf-L6 & $26.7451(1)$ & $19130.9(2)$ & $2.214(2)$ & $11.171(4)$ \\
\hline Hf-L7 & $29.2116(9)$ & $24926.7(20)$ & $2.194(12)$ & $12.959(17)$ \\
\hline Zr-L8 & $29.8884(3)$ & $26699.8(8)$ & $2.199(6)$ & $13.419(8)$ \\
\hline
\end{tabular}

modulator. For the longer linkers, the incorporation of smaller hydrophobic amino acids into MOF synthesis results in significantly improved crystallinity. L-Proline is again found to be the most effective modulator, although the synthesis of Hf-L8 seems to benefit most from inclusion of L-tryptophan.

Single crystals of Hf-L6 suitable for X-ray diffraction were isolated from solvothermal syntheses in DMF at $120^{\circ} \mathrm{C}$ for $24 \mathrm{~h}$ with five equivalents of L-proline and one equivalent of $\mathrm{HCl}$ as modulators (see ESI, Section S9†). With small modifications in the synthesis procedure, it was possible to isolate single crystals of Hf-L7, using three equivalents of L-proline and one of $\mathrm{HCl}$ as modulators in a solvothermal synthesis in DMF at $100{ }^{\circ} \mathrm{C}$ for 48 h. Both MOFs are, as expected, isostructural to their zirconium analogues, with a comparison of some pertinent crystallographic details from the five structures (Table 1) illustrating the similarities between the $\mathrm{Zr}$ and $\mathrm{Hf}$ analogues. We have as yet been unable to isolate single crystals of Hf-L8, likely due to the tendency to form spherical microcrystalline architectures when modulated by L-proline in a manner similar to Zr-L8 (see ESI, Section S8†).

With a range of analogous $\mathrm{Zr}$ and Hf MOFs in hand, it was possible to examine their physical properties and similarities. Previous reports of the monocarboxylic acid modulation of $\mathbf{Z r}$ L6 have described ${ }^{6 \boldsymbol{d}, 16 a, 19,28}$ incorporation of acid modulators into the structure, postulated to be at defect or surface inclusion sites, and so ${ }^{1} \mathrm{H}$ NMR spectra of acid-digested samples of $\mathrm{Zr}$ and Hf MOFs of L5-L8 were examined for evidence of L-proline incorporation (see ESI, Section S10†). Only Zr-L5 and Hf-L5 appeared to contain any appreciable quantities of L-proline or its breakdown products, which may be due to their smaller pore size than the other MOFs, although their incorporation at defects or as surface ligands cannot be ruled out. Thermogravimetric analysis (TGA) of the MOFs confirms that the Hf MOFs retain the thermal stability of their $\mathrm{Zr}$ analogues (see ESI, Section S11 $\dagger$ ), while an additional mass loss event at $350-450{ }^{\circ} \mathrm{C}$ for Zr-L5 and Hf-L5 is observed that can be ascribed to removal of incorporated L-proline, with a modulator content of $10 \% \mathrm{w} / \mathrm{w}$ calculated for the two analogous MOFs. Subtle changes in TGA profiles are evident as the ligand is changed for both the $\mathrm{Zr}$ and Hf MOFs, indicating that the organic linker has a role to play in the thermal stability of the MOFs.

$\mathrm{N}_{2}$ adsorption isotherms (see ESI, Section S12 $\dagger$ ) carried out at $77 \mathrm{~K}$ on the L-proline modulated materials show the high surface areas expected for highly crystalline MOFs (Fig. 6). In the case where the $\mathrm{Zr}$ MOFs have been reported previously, ${ }^{9 b, 16 b, 24,30}$ the BET surface areas closely correlate to those

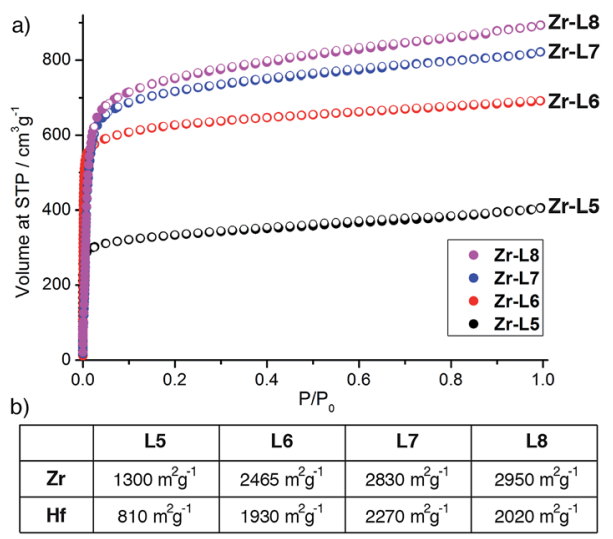

Fig. 6 (a) $\mathrm{N}_{2}$ adsorption isotherms (77 K) for Zr-L5, Zr-L6, Zr-L7 and $\mathrm{Zr}$-L8. (b) BET surface areas for all $\mathrm{Zr}$ and Hf MOFs, as derived from their $\mathrm{N}_{2}$ adsorption isotherms.

previously measured; $1300 \mathrm{~m}^{2} \mathrm{~g}^{-1}$ (Zr-L5), $2465 \mathrm{~m}^{2} \mathrm{~g}^{-1}$ (Zr-L6), $2830 \mathrm{~m}^{2} \mathrm{~g}^{-1}$ (Zr-L7) and $2950 \mathrm{~m}^{2} \mathrm{~g}^{-1}$ (Zr-L8). The value for Zr-L5 is slightly lower than reported previously by Kaskel et al. $\left(1400 \mathrm{~m}^{2} \mathrm{~g}^{-1}\right)^{9 b}$ which may be a consequence of the presence of L-proline, as indicated by ${ }^{1} \mathrm{H}$ NMR spectroscopy and TGA. Pore size distributions calculated using QSDFT show an increase in major pore diameter in concert with the increase in linker length. The Hf analogues show decreased gravimetric surface areas in line with the increased mass of $\mathrm{Hf}$ compared to $\mathrm{Zr}$, however, the surface areas of Hf-L5 $\left(810 \mathrm{~m}^{2} \mathrm{~g}^{-1}\right)$ and Hf-L8 $\left(2020 \mathrm{~m}^{2} \mathrm{~g}^{-1}\right)$ are slightly lower than expected based on this mass increase. Interestingly, both of these MOFs display spherical microcrystalline aggregates under SEM imaging, whereas all other MOFs formed either discrete single crystals or films, but the effect of morphology on accessible surface area has not been further investigated. The BET surface areas of Hf-L6 $\left(1930 \mathrm{~m}^{2} \mathrm{~g}^{-1}\right)$ and Hf-L7 $\left(2270 \mathrm{~m}^{2} \mathrm{~g}^{-1}\right)$ correlate closely to their corresponding $\mathrm{Zr}$ analogues.

\section{Conclusions}

To conclude, we report that amino acids, in concert with $\mathrm{HCl}$, can effectively modulate the syntheses of zirconium and hafnium MOFs, enhancing crystallinity and ultimately resulting in the isolation of previously inaccessible MOFs, many as single crystals suitable for X-ray diffraction. Whilst a range of amino acids have been investigated, the greatest success has been achieved using L-proline, which is required in as little as four 
equivalents - a great reduction when compared with the large excess of modulator routinely required for single crystal synthesis (see ESI, Section S13†) - to offer unprecedented access to defect free single crystals of a number of UiO-66 topology MOFs of both $\mathrm{Zr}$ and Hf. ${ }^{33}$ The ability to reliably synthesise $\mathrm{Zr}$ and Hf MOFs simply and efficiently has allowed us to compare the properties of the analogous materials. We have found that the change in metal has little effect on thermal stability, and $\mathrm{N}_{2}$ adsorption isotherms show the MOFs have the high surface areas expected from materials of excellent crystallinity. Access to single crystals of $\mathrm{Zr}$ MOFs has already facilitated detailed investigations of their mechanical properties by high pressure single crystal X-ray diffraction and nanoindentation, ${ }^{\mathbf{2 1}}$ and also single-crystal to single-crystal post-synthetic modification. ${ }^{14 e}$ We expect that adoption of amino acid modulated synthetic protocols, either solvothermally or microwave heated, will result in the improved synthesis of other $\mathrm{Zr}$ and Hf MOFs, and we are currently attempting to elucidate the mechanism of modulation while extending the protocol to MOFs linked by other metals.

\section{Acknowledgements}

RSF thanks the Royal Society for receipt of a University Research Fellowship and the University of Glasgow for funding. The research was supported at the University of Glasgow by EPSRC (EP/L004461/1). CLH thanks the EPSRC and the University of Edinburgh for a studentship. CAM acknowledges the UK CarParrinello consortium (EP/K01465X/1) for access to the high performance computing resource ARCHER, as managed by the Edinburgh Parallel Computing Service. The authors gratefully acknowledge pump-priming funding from the EPSRC Directed Assembly Network (PP 1405 03). We thank Mr Jim Gallagher at the University of Glasgow for assistance with SEM. The data which underpin this work are available at http:/dx.doi.org/ 10.5525/gla.research-data.251. CCDC 1442841-1442842† contain the supplementary crystallographic data for this paper.

\section{References}

1 H. Furukawa, K. E. Cordova, M. O'Keeffe and O. M. Yaghi, Science, 2013, 341, 6149.

2 (a) D. Britt, D. Tranchemontagne and O. M. Yaghi, Proc. Natl. Acad. Sci. U. S. A., 2008, 105, 11623-11627; (b) S. Ma and H.-C. Zhou, Chem. Commun., 2010, 46, 44-53; (c) L. J. Murray, M. Dinca and J. R. Long, Chem. Soc. Rev., 2009, 38, 1294-1314; (d) K. Sumida, D. L. Rogow, J. A. Mason, T. M. McDonald, E. D. Bloch, Z. R. Herm, T.-H. Bae and J. R. Long, Chem. Rev., 2012, 112, 724-781.

3 (a) A. C. McKinlay, R. E. Morris, P. Horcajada, G. Férey, R. Gref, P. Couvreur and C. Serre, Angew. Chem., Int. Ed., 2010, 49, 6260-6266; (b) P. Horcajada, T. Chalati, C. Serre, B. Gillet, C. Sebrie, T. Baati, J. F. Eubank, D. Heurtaux, P. Clayette, C. Kreuz, J.-S. Chang, Y. K. Hwang, V. Marsaud, P.-N. Bories, L. Cynober, S. Gil, G. Férey, P. Couvreur and R. Gref, Nat. Mater., 2010, 9, 172-178; (c) P. Horcajada, C. Serre, G. Maurin, N. A. Ramsahye, F. Balas, M. Vallet-
Regí, M. Sebban, F. Taulelle and G. Férey, J. Am. Chem. Soc., 2008, 130, 6774-6780.

4 (a) J. Lee, O. K. Farha, J. Roberts, K. A. Scheidt, S. T. Nguyen and J. T. Hupp, Chem. Soc. Rev., 2009, 38, 1450-1459; (b) M. Yoon, R. Srirambalaji and K. Kim, Chem. Rev., 2012, 112, 1196-1231; (c) A. Corma, H. García and F. X. Llabrés i Xamena, Chem. Rev., 2010, 110, 4606-4655.

5 (a) R. J. Marshall, T. Richards, C. Hobday, C. F. Murphie, C. Wilson, S. A. Moggach, T. D. Bennett and R. S. Forgan, Dalton Trans., 2016, DOI: 10.1039/C1035DT03178H; (b) J. H. Cavka, S. Jakobsen, U. Olsbye, N. Guillou, C. Lamberti, S. Bordiga and K. P. Lillerud, J. Am. Chem. Soc., 2008, 130, 13850-13851; (c) J. B. DeCoste, G. W. Peterson, H. Jasuja, T. G. Glover, Y.-G. Huang and K. S. Walton, J. Mater. Chem. A, 2013, 1, 5642-5650; (d) M. Kandiah, M. H. Nilsen, S. Usseglio, S. Jakobsen, U. Olsbye, M. Tilset, C. Larabi, E. A. Quadrelli, F. Bonino and K. P. Lillerud, Chem. Mater., 2010, 22, 6632-6640; (e) J. E. Mondloch, M. J. Katz, N. Planas, D. Semrouni, L. Gagliardi, J. T. Hupp and O. K. Farha, Chem. Commun., 2014, 50, 8944-8946; (f) B. van de Voorde, I. Stassen, B. Bueken, F. Vermoortele, D. De Vos, R. Ameloot, J.-C. Tan and T. D. Bennett, J. Mater. Chem. A, 2015, 3, 1737-1742; (g) H. Wu, T. Yildirim and W. Zhou, J. Phys. Chem. Lett., 2013, 4, 925-930; (h) L.-M. Yang, E. Ganz, S. Svelle and M. Tilset, J. Mater. Chem. C, 2014, 2, 7111-7125; (i) C. Hobday, R. J. Marshall, C. F. Murphie, T. Richards, D. Allan, T. Duren, F.-X. Coudert, R. S. Forgan, C. A. Morrison, S. A. Moggach and T. D. Bennett, Angew. Chem., Int. Ed., 2016, 55, 2401-2405.

6 (a) R. Wang, Z. Wang, Y. Xu, F. Dai, L. Zhang and D. Sun, Inorg. Chem., 2014, 53, 7086-7088; (b) H. Furukawa, F. Gándara, Y.-B. Zhang, J. Jiang, W. L. Queen, M. R. Hudson and O. M. Yaghi, J. Am. Chem. Soc., 2014, 136, 4369-4381; (c) V. Guillerm, F. Ragon, M. Dan-Hardi, T. Devic, M. Vishnuvarthan, B. Campo, A. Vimont, G. Clet, Q. Yang, G. Maurin, G. Férey, A. Vittadini, S. Gross and C. Serre, Angew. Chem., Int. Ed., 2012, 51, 9267-9271; (d) A. Schaate, P. Roy, A. Godt, J. Lippke, F. Waltz, M. Wiebcke and P. Behrens, Chem.-Eur. J., 2011, 17, 6643-6651; (e) A. Schaate, P. Roy, T. Preuße, S. J. Lohmeier, A. Godt and P. Behrens, Chem.-Eur. J., 2011, 17, 9320-9325; (f) W. Morris, B. Volosskiy, S. Demir, F. Gándara, P. L. McGrier, H. Furukawa, D. Cascio, J. F. Stoddart and O. M. Yaghi, Inorg. Chem., 2012, 51, 6443-6445; $(g)$ T. C. Wang, W. Bury, D. A. Gómez-Gualdrón, N. A. Vermeulen, J. E. Mondloch, P. Deria, K. Zhang, P. Z. Moghadam, A. A. Sarjeant, R. Q. Snurr, J. F. Stoddart, J. T. Hupp and O. K. Farha, J. Am. Chem. Soc., 2015, 137, 3585-3591; (h) D. Feng, Z.-Y. Gu, J.-R. Li, H.-L. Jiang, Z. Wei and H.-C. Zhou, Angew. Chem., Int. Ed., 2012, 51, 10307-10310; (i) D. Feng, H.-L. Jiang, Y.-P. Chen, Z.-Y. Gu, Z. Wei and H.-C. Zhou, Inorg. Chem., 2013, 52, 1266112667; (j) D. Feng, K. Wang, J. Su, T.-F. Liu, J. Park, Z. Wei, M. Bosch, A. Yakovenko, X. Zou and H.-C. Zhou, Angew. Chem., Int. Ed., 2015, 54, 149-154; (k) J. E. Mondloch, W. Bury, D. Fairen-Jimenez, S. Kwon, E. J. DeMarco, 
M. H. Weston, A. A. Sarjeant, S. T. Nguyen, P. C. Stair, R. Q. Snurr, O. K. Farha and J. T. Hupp, J. Am. Chem. Soc., 2013, 135, 10294-10297; ( $l$ ) W. Liang, H. Chevreau, F. Ragon, P. D. Southon, V. K. Peterson and D. M. D'Alessandro, CrystEngComm, 2014, 16, 6530-6533.

7 (a) P. W. Siu, Z. J. Brown, O. K. Farha, J. T. Hupp and K. A. Scheidt, Chem. Commun., 2013, 49, 10920-10922; (b) S. J. Garibay and S. M. Cohen, Chem. Commun., 2010, 46, 7700-7702; (c) H.-L. Jiang, D. Feng, T.-F. Liu, J.-R. Li and H.-C. Zhou, J. Am. Chem. Soc., 2012, 134, 14690-14693; (d) B. Wang, H. Huang, X.-L. Lv, Y. Xie, M. Li and J.-R. Li, Inorg. Chem., 2014, 53, 9254-9259; (e) K. Manna, T. Zhang and W. Lin, J. Am. Chem. Soc., 2014, 136, 6566-6569; $(f)$ H. Fei and S. M. Cohen, Chem. Commun., 2014, 50, 48104812 .

8 S. Jakobsen, D. Gianolio, D. S. Wragg, M. H. Nilsen, H. Emerich, S. Bordiga, C. Lamberti, U. Olsbye, M. Tilset and K. P. Lillerud, Phys. Rev. B: Condens. Matter Mater. Phys., 2012, 86, 125429.

9 (a) V. Bon, I. Senkovska, I. A. Baburin and S. Kaskel, Cryst. Growth Des., 2013, 13, 1231-1237; (b) V. Bon, I. Senkovska, M. S. Weiss and S. Kaskel, CrystEngComm, 2013, 15, 95729577; (c) V. Bon, V. Senkovskyy, I. Senkovska and S. Kaskel, Chem. Commun., 2012, 48, 8407-8409; (d) M. J. Cliffe, W. Wan, X. Zou, P. A. Chater, A. K. Kleppe, M. G. Tucker, H. Wilhelm, N. P. Funnell, F.-X. Coudert and A. L. Goodwin, Nat. Commun., 2014, 5, 4176; (e) M. Zhang, Y.-P. Chen, M. Bosch, T. Gentle, K. Wang, D. Feng, Z. U. Wang and H.-C. Zhou, Angew. Chem., Int. Ed., 2014, 53, 815-818; $(f)$ P. Xydias, I. Spanopoulos, E. Klontzas, G. E. Froudakis and P. N. Trikalitis, Inorg. Chem., 2014, 53, 679-681.

10 (a) K. Lu, C. He and W. Lin, J. Am. Chem. Soc., 2014, 136, 16712-16715; (b) K. E. deKrafft, W. S. Boyle, L. M. Burk, O. Z. Zhou and W. Lin, J. Mater. Chem., 2012, 22, 1813918144.

11 J. Zheng, M. Wu, F. Jiang, W. Su and M. Hong, Chem. Sci., 2015, 6, 3466-3470.

12 M. H. Beyzavi, R. C. Klet, S. Tussupbayev, J. Borycz, N. A. Vermeulen, C. J. Cramer, J. F. Stoddart, J. T. Hupp and O. K. Farha, J. Am. Chem. Soc., 2014, 136, 15861-15864.

13 C. V. McGuire and R. S. Forgan, Chem. Commun., 2015, 51, 5199-5217.

14 (a) M. I. Gonzalez, E. D. Bloch, J. A. Mason, S. J. Teat and J. R. Long, Inorg. Chem., 2015, 54, 2995-3005; (b) V. Guillerm, S. Gross, C. Serre, T. Devic, M. Bauer and G. Férey, Chem. Commun., 2010, 46, 767-769; (c) B. Li, B. Gui, G. Hu, D. Yuan and C. Wang, Inorg. Chem., 2015, 54, 5139-5141; (d) L. Li, S. Tang, C. Wang, X. Lv, M. Jiang, H. Wu and X. Zhao, Chem. Commun., 2014, 50, 2304-2307; (e) R. J. Marshall, S. L. Griffin, C. Wilson and R. S. Forgan, J. Am. Chem. Soc., 2015, 137, 9527-9530; (f) C. A. Trickett, K. J. Gagnon, S. Lee, F. Gándara, H.-B. Bürgi and O. M. Yaghi, Angew. Chem., Int. Ed., 2015, 54, 11162-11167; (g) G. Wißmann, A. Schaate, S. Lilienthal, I. Bremer, A. M. Schneider and P. Behrens, Microporous Mesoporous Mater., 2012, 152, 64-70.
15 G. Zahn, P. Zerner, J. Lippke, F. L. Kempf, S. Lilienthal, C. A. Schroder, A. M. Schneider and P. Behrens, CrystEngComm, 2014, 16, 9198-9207.

16 (a) O. V. Gutov, M. G. Hevia, E. C. Escudero-Adán and A. Shafir, Inorg. Chem., 2015, 54, 8396-8400; (b) M. J. Katz, Z. J. Brown, Y. J. Colon, P. W. Siu, K. A. Scheidt, R. Q. Snurr, J. T. Hupp and O. K. Farha, Chem. Commun., 2013, 49, 9449-9451; (c) G. C. Shearer, S. Chavan, J. Ethiraj, J. G. Vitillo, S. Svelle, U. Olsbye, C. Lamberti, S. Bordiga and K. P. Lillerud, Chem. Mater., 2014, 26, 4068-4071; (d) L. Valenzano, B. Civalleri, S. Chavan, S. Bordiga, M. H. Nilsen, S. Jakobsen, K. P. Lillerud and C. Lamberti, Chem. Mater., 2011, 23, 1700-1718.

17 H. Wu, Y. S. Chua, V. Krungleviciute, M. Tyagi, P. Chen, T. Yildirim and W. Zhou, J. Am. Chem. Soc., 2013, 135, 10525-10532.

18 J. M. Taylor, S. Dekura, R. Ikeda and H. Kitagawa, Chem. Mater., 2015, 27, 2286-2289.

19 F. Vermoortele, B. Bueken, G. Le Bars, B. van de Voorde, M. Vandichel, K. Houthoofd, A. Vimont, M. Daturi, M. Waroquier, V. van Speybroeck, C. Kirschhock and D. E. De Vos, J. Am. Chem. Soc., 2013, 135, 11465-11468.

20 F. Ragon, P. Horcajada, H. Chevreau, Y. K. Hwang, U. H. Lee, S. R. Miller, T. Devic, J.-S. Chang and C. Serre, Inorg. Chem., 2014, 53, 2491-2500.

21 The synthesis and crystallographic characterisation of Zr-L6 and Zr-L7 were recently reported by us in a preliminary communication concerning their mechanical properties (see ref. 5i). The synthesis and crystallographic characterisation of $\mathbf{Z r}-\mathbf{L 8}$ were recently reported by us in a communication concerning the effect of post-synthetic modification on mechanical properties (see ref. $5 a$ ).

22 During the courseof our study, it became apparent that Zr-L8 was unstable in the presence of methanol, which has previously been observed for certain MOFs of the UiO-66 topology (see ref. $5 c$ ). To avoid sample degradation, acetone replaced methanol in the work up of all MOFs containing L8 (see ESI $\dagger$ ).

$23 \mathrm{HCl}$ has previously been reported to enhance the synthesis of members of the UiO-66 series when incorporated in large quantities into solvothermal syntheses (see ref. $16 b$ and 20). In our hands, syntheses of Zr-MOFs of ligands L5-L8 with the addition of only one equivalent of $\mathrm{HCl}$ did not give reproducible results, particularly in the synthesis of Zr-L6 (UiO-67) with some products showing good crystallinity and others being completely amorphous. This observation correlates with previous work by Behrens, et al., who reported that addition of $\mathrm{HCl}$ did not improve their syntheses of UiO-67 (see ref. 6d).

24 A. Schaate, S. Dühnen, G. Platz, S. Lilienthal, A. M. Schneider and P. Behrens, Eur. J. Inorg. Chem., 2012, 2012, 790-796.

25 (a) Y. Li, Y. Liu, W. Gao, L. Zhang, W. Liu, J. Lu, Z. Wang and Y.-J. Deng, CrystEngComm, 2014, 16, 7037-7042; (b) M. Taddei, P. V. Dau, S. M. Cohen, M. Ranocchiari, J. A. van Bokhoven, F. Costantino, S. Sabatini and R. Vivani, Dalton Trans., 2015, 44, 14019-14026. 
26 G. Nickerl, M. Leistner, S. Helten, V. Bon, I. Senkovska and S. Kaskel, Inorg. Chem. Front., 2014, 1, 325-330.

27 S. Øien, D. Wragg, H. Reinsch, S. Svelle, S. Bordiga, C. Lamberti and K. P. Lillerud, Cryst. Growth Des., 2014, 14, 5370-5372.

28 N. Ko, J. Hong, S. Sung, K. E. Cordova, H. J. Park, J. K. Yang and J. Kim, Dalton Trans., 2015, 44, 2047-2051.

29 Zr-L6 crystallises in the $F m \overline{3} m$ cubic space group, with a unit cell edge $a=26.8564(5) \AA ̊$ for our DMF-solvated sample, a value very similar to the reported parameters for (i) a fully DMF solvated sample, where $a=26.896(3) \AA$ (see ref. 26), a second DMF solvated sample, where $a=$ 26.783(3) A (see ref. 28), a third DMF solvated sample, where $a=26.812(2) \AA$ (see ref. 16a), (iv) a partially desolvated crystal, where $a=26.8809(3) \AA$ (see ref. 27), and (v) a powdered sample, where $a=26.8499(15) \AA$ (see ref. $6 d$ ).
30 L. T. M. Hoang, L. H. Ngo, H. L. Nguyen, H. T. H. Nguyen, C. K. Nguyen, B. T. Nguyen, Q. T. Ton, H. K. D. Nguyen, K. E. Cordova and T. Truong, Chem. Commun., 2015, 51, 17132-17135.

31 Our experimental values for Zr-L7 compare well to previously reported values of $a=29.4227(4) \AA$ and $a=29.4433(4) \AA$ for powder and single crystal examples, respectively (see ref. 24) and $a=29.3994(2) \AA$ in the space group $F m \overline{3} m$; for a powder sample (see ref. 30).

32 L-Arginine was not used in these evaluations as it showed no modulating effect for the Zr-MOFs and generally produced tar-like materials rather than powder.

33 While some modulators that are usually utilised in large amounts during synthesis of $\mathrm{Zr}$ MOFs are available at very low cost (e.g. formic and acetic acid) our highly efficient amino acid modulated syntheses dramatically cut down on waste and do not routinely induce defects in the MOFs. 